\title{
Medium Domination Number in Linear Polyacene
}

\author{
S.Venkatesan, M.Raji, G.Jayalalitha
}

\begin{abstract}
Let $G(V, E)$ be a finite, connected graph. The set of vertices and edges of $G$ are denoted by $V=V(G)$ and $E=E(G)$ respectively. In such a Molecular graph, vertices represent atoms and edges represent bonds. In this paper, it obtains Medium Domination Number in the Molecular Graph of Organic Compounds and proves by induction method.

Keywords: Dominating set, Medium Domination Number, Internally Disjoint Path, Molecular Graph.

AMS Subject Classification: 05C69
\end{abstract}

\section{INTRODUCTION}

For a Molecular Graph, vertices denote atoms and edges denote bonds[8]. The structure of Molecular graph may be connected, undirected, loop less graph. In a graph, each vertex is able to protect every vertex in its neighborhood and in domination every vertex needs to be prevented. In such case, it is required to inspect the number of vertices those are dominating each pair of vertices. The summation of vertices that dominate every set of vertices and mean value of this is defined as the medium domination number of a graph[2].

Chemical graph theory is based on Mathematics which mixes graph concepts and chemistry. Graph theory is applied to mathematically model molecules so as to achieve insight into the physical properties of those chemical compounds. Chemical graph is a suitable model for any real or abstracted chemical system. Let L (h) be the Linear Polyacene with $h$ hexagons. Knowing that If $\mathrm{L}$ (h) is a Linear Polyacene with $h$ hexagons, then $\mathrm{n}(\mathrm{L}(\mathrm{h}))=4 \mathrm{~h}+2$.If $\mathrm{L}(\mathrm{h})$ is a Linear Polyacene with $\mathrm{h}$ hexagons , then $Y(L(h))=h+1$. where No. of vertices is $n(L(h))$ and Domination No. of Linear Polyacene in is $\mathrm{Y}(\mathrm{L}(\mathrm{h}))[6]$.In this paper, all graphs are considered as a finite, undirected graph without loops and multiple edges. here it obtains the Medium Domination number in the molecular graph of organic compounds using Induction method.

\section{PRELIMINARIES}

\subsection{GRAPH [1]:}

A graph $\mathrm{G}=(\mathrm{V}, \mathrm{E})$ consists of a nonempty set $\mathrm{V}$ of vertices and a set $\mathrm{E}$ of edges.

\subsection{DOMINATING SET [2]:}

A set $D$ in a graph ' $G$ ' is a dominating set if each vertex is either in $\mathrm{D}$ or adjacent to a vertex in $\mathrm{D}$.

Revised Manuscript Received on July 18, 2019.

S.Venkatesan, Research Scholar,Department of Mathematics, VELS Institute of Science, Technology \& Advanced Studies, Chennai, Tamil Nadu , India.

M.Raji, Research Scholar,Department of Mathematics, VELS Institute of Science, Technology \& Advanced Studies, Chennai, Tamil Nadu , India.

G.Jayalalitha, Professor, Department of Mathematics, VELS Institute of Science, Technology \& Advanced Studies, Chennai, Tamil Nadu , India. (rajialagumurugan@gmail.com), (ragaji94@yahoo.com)

\section{$2.3 \mathrm{~K}-\mathrm{CONNECTED[2]}$}

Two vertices $u$ and $v$ in a graph $G$ are said to be $\mathbf{k}$ connected if there are $\mathrm{k}$ or more pairwise internally disjoint paths between them.

\subsection{DOMINATES EACH OTHER [2,3]}

If $\mathrm{u}$ and $\mathrm{v}$ are adjacent in a graph $\mathrm{G}$, they dominates each other, then at least

$\operatorname{dom}(\mathbf{u}, \mathbf{v})=\mathbf{1}$ where $\operatorname{dom}(\mathrm{u}, \mathrm{v})$ denotes the number of vertices that dominate both of $\mathrm{u} \& \mathrm{v}$.

\subsection{TOTAL DOMINATING VERTICES $(T D V(G))$ [2,3]}

The summation of vertices that dominate every set of two vertices is defined as

$$
\operatorname{TDV}(G)=\sum_{\forall u, v \in V(G)} \operatorname{dom}(u, v)
$$

\subsection{MEDIUM DOMINATION NUMBER $(M D(G))[2,3]$}

If the order of $\mathrm{G}$ is $\mathrm{n}$, then Medium Domination Number is defined as

$M D(G)=\frac{2 \operatorname{TDV}(G)}{n(n-1)}$.

\section{MOLECULAR GRAPH OF ORGANIC COMPOUNDS \& RESULTS}

In this section, it obtains Medium Domination Number for the Molecular Graph of Benzene, Naphthalene and Anthracene and also obtains Medium Domination Number Of Linear Polyacene $\boldsymbol{L}_{\boldsymbol{h}}$ is $\operatorname{MD}\left(\boldsymbol{L}_{\boldsymbol{h}}\right)=2 \sum_{k=1}^{h} \frac{13 k-1}{(4 k+2)(4 k+1)}$ .Using Theorem $\operatorname{dom}(\boldsymbol{u}, \boldsymbol{v})=\boldsymbol{k}_{1}(\boldsymbol{u}, \boldsymbol{v})+\boldsymbol{k}_{2}(\boldsymbol{u}, \boldsymbol{v})$ where $k_{1}$ and $k_{2}$ denote the number of $u-v$ internally disjoint paths of length 1 and 2 respectively, for calculating dominates each other of joining vertices $u$ and $v[2]$.

\subsection{MOLECULAR GRAPH OF BENZENE}

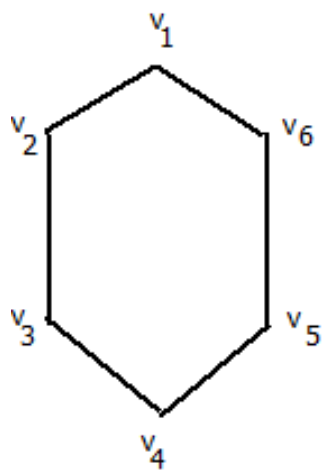

Figure 1.Molecular Graph of $L_{1}$ (Benzene)

\section{Published By:}


In the Molecular Graph of Benzene ,from figure1, we get $\operatorname{dom}\left(\mathrm{v}_{1}, \mathrm{v}_{2}\right)=1 ; \operatorname{dom}\left(\mathrm{v}_{1}, \mathrm{v}_{3}\right)=1 ; \operatorname{dom}\left(\mathrm{v}_{1}, \mathrm{v}_{4}\right)=0 ; \operatorname{dom}$ $\left(\mathrm{v}_{1}, \mathrm{v}_{5}\right)=1 ; \operatorname{dom}\left(\mathrm{v}_{1}, \mathrm{v}_{6}\right)=1 ; \operatorname{dom}\left(\mathrm{v}_{2}, \mathrm{v}_{3}\right)=1 ;$

$\operatorname{dom}\left(\mathrm{v}_{2}, \mathrm{v}_{4}\right)=1 ; \operatorname{dom}\left(\mathrm{v}_{2}, \mathrm{v}_{5}\right)=0 ; \operatorname{dom}\left(\mathrm{v}_{2}, \mathrm{v}_{6}\right)=1 ; \operatorname{dom}$ $\left(\mathrm{v}_{3}, \mathrm{v}_{4}\right)=1 ; \operatorname{dom}\left(\mathrm{v}_{3}, \mathrm{v}_{5}\right)=1 ; \operatorname{dom}\left(\mathrm{v}_{3}, \mathrm{v}_{6}\right)=0$;

$\operatorname{dom}\left(\mathrm{v}_{4}, \mathrm{v}_{5}\right)=1 ; \operatorname{dom}\left(\mathrm{v}_{4}, \mathrm{v}_{6}\right)=1 ; \operatorname{dom}\left(\mathrm{v}_{5}, \mathrm{v}_{6}\right)=1$.

$\operatorname{TDV}(G)=12$.

Medium Domination Number $=\frac{2(12)}{6(5)}=\frac{4}{5}$.

\subsection{MOLECULAR GRAPH OF NAPATHALENE[5]}

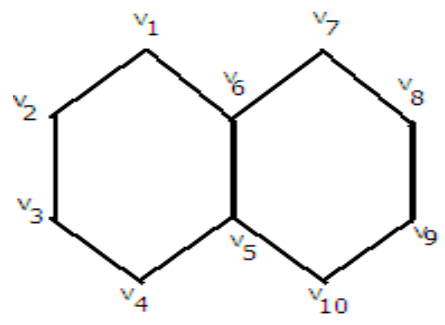

Figure 2.Molecular Graph of Naphthalene

In the Molecular Graph of Naphthalene, from figure 2, we get

$\operatorname{dom}\left(\mathrm{v}_{1}, \mathrm{v}_{2}\right)=1 ; \operatorname{dom}\left(\mathrm{v}_{1}, \mathrm{v}_{3}\right)=1 ; \operatorname{dom}\left(\mathrm{v}_{1}, \mathrm{v}_{4}\right)=0 ; \operatorname{dom}$

$\left(\mathrm{v}_{1}, \mathrm{v}_{5}\right)=1 ; \operatorname{dom}\left(\mathrm{v}_{1}, \mathrm{v}_{6}\right)=1 ; \operatorname{dom}\left(\mathrm{v}_{1}, \mathrm{v}_{7}\right)=1$

$\operatorname{dom}\left(\mathrm{v}_{1}, \mathrm{v}_{8}\right)=0 ; \operatorname{dom}\left(\mathrm{v}_{1}, \mathrm{v}_{9}\right)=0 ; \operatorname{dom}\left(\mathrm{v}_{1}, \mathrm{v}_{10}\right)=0 ; \operatorname{dom}$ $\left(\mathrm{v}_{2}, \mathrm{v}_{3}\right)=1 ; \operatorname{dom}\left(\mathrm{v}_{2}, \mathrm{v}_{4}\right)=1 ; \operatorname{dom}\left(\mathrm{v}_{2}, \mathrm{v}_{5}\right)=0 ;$

$\operatorname{dom}\left(\mathrm{v}_{2}, \mathrm{v}_{6}\right)=1 ; \operatorname{dom}\left(\mathrm{v}_{2}, \mathrm{v}_{7}\right)=0 ; \operatorname{dom}\left(\mathrm{v}_{2}, \mathrm{v}_{8}\right)=0 ; \operatorname{dom}$

$\left(\mathrm{v}_{2}, \mathrm{v}_{9}\right)=0 ; \operatorname{dom}\left(\mathrm{v}_{2}, \mathrm{v}_{10}\right)=0 ; \operatorname{dom}\left(\mathrm{v}_{3}, \mathrm{v}_{4}\right)=1 ;$

$\operatorname{dom}\left(\mathrm{v}_{3}, \mathrm{v}_{5}\right)=1 ; \operatorname{dom}\left(\mathrm{v}_{3}, \mathrm{v}_{6}\right)=0 ; \operatorname{dom}\left(\mathrm{v}_{3}, \mathrm{v}_{7}\right)=0 ; \operatorname{dom}$

$\left(\mathrm{v}_{3}, \mathrm{v}_{8}\right)=0 ; \operatorname{dom}\left(\mathrm{v}_{3}, \mathrm{v}_{9}\right)=0 ; \operatorname{dom}\left(\mathrm{v}_{3}, \mathrm{v}_{10}\right)=0 ;$

$\operatorname{dom}\left(\mathrm{v}_{4}, \mathrm{v}_{5}\right)=1 ; \operatorname{dom}\left(\mathrm{v}_{4}, \mathrm{v}_{6}\right)=1 ; \operatorname{dom}\left(\mathrm{v}_{4}, \mathrm{v}_{7}\right)=0 ; \operatorname{dom}$

$\left(\mathrm{v}_{4}, \mathrm{v}_{8}\right)=0 ; \operatorname{dom}\left(\mathrm{v}_{4}, \mathrm{v}_{9}\right)=0 ; \operatorname{dom}\left(\mathrm{v}_{4}, \mathrm{v}_{10}\right)=1 ;$

$\operatorname{dom}\left(\mathrm{v}_{5}, \mathrm{v}_{6}\right)=1 ; \operatorname{dom}\left(\mathrm{v}_{5}, \mathrm{v}_{7}\right)=1 ; \operatorname{dom}\left(\mathrm{v}_{5}, \mathrm{v}_{8}\right)=0 ; \operatorname{dom}$

$\left(\mathrm{v}_{5}, \mathrm{v}_{9}\right)=1 ; \operatorname{dom}\left(\mathrm{v}_{5}, \mathrm{v}_{10}\right)=1 ; \operatorname{dom}\left(\mathrm{v}_{6}, \mathrm{v}_{7}\right)=1 ;$

$\operatorname{dom}\left(\mathrm{v}_{6}, \mathrm{v}_{8}\right)=1 ; \operatorname{dom}\left(\mathrm{v}_{6}, \mathrm{v}_{9}\right)=0 ; \operatorname{dom}\left(\mathrm{v}_{6}, \mathrm{v}_{10}\right)=1 ; \operatorname{dom}$

$\left(\mathrm{v}_{7}, \mathrm{v}_{8}\right)=1 ; \operatorname{dom}\left(\mathrm{v}_{7}, \mathrm{v}_{9}\right)=1 ; \operatorname{dom}\left(\mathrm{v}_{7}, \mathrm{v}_{10}\right)=0 ;$

$\operatorname{dom}\left(\mathrm{v}_{8}, \mathrm{v}_{9}\right)=1 ; \operatorname{dom}\left(\mathrm{v}_{8}, \mathrm{v}_{10}\right)=1 ; \operatorname{dom}\left(\mathrm{v}_{9}, \mathrm{v}_{10}\right)=1$.

$\operatorname{TDV}(G)=25$.

Medium Domination Number $=\frac{2(25)}{10(9)}=\frac{5}{9}$.

\subsection{MOLECULAR GRAPH OF ANTHRACENE [4]:}

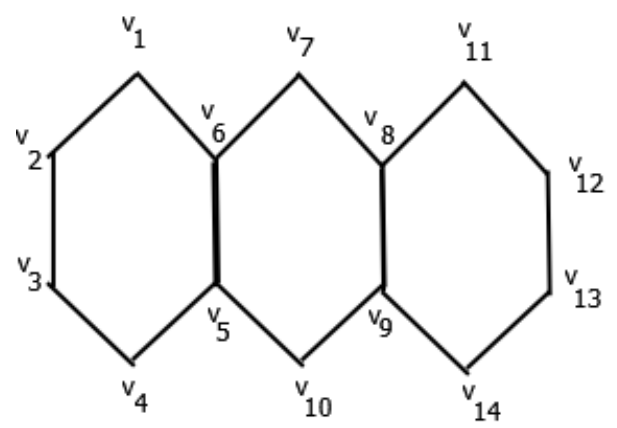

Figure 3.Molecular Graph of Anthracene

In the Molecular Graph of Anthracene, from figure 3,we get

$\operatorname{dom}\left(\mathrm{v}_{1}, \mathrm{v}_{2}\right)=1 ; \operatorname{dom}\left(\mathrm{v}_{1}, \mathrm{v}_{3}\right)=1 ; \operatorname{dom}\left(\mathrm{v}_{1}, \mathrm{v}_{4}\right)=0 ; \operatorname{dom}$

$\left(\mathrm{v}_{1}, \mathrm{v}_{5}\right)=1 ; \operatorname{dom}\left(\mathrm{v}_{1}, \mathrm{v}_{6}\right)=1 ; \operatorname{dom}\left(\mathrm{v}_{1}, \mathrm{v}_{7}\right)=1$; $\operatorname{dom}\left(\mathrm{v}_{1}, \mathrm{v}_{8}\right)=0 ; \operatorname{dom}\left(\mathrm{v}_{1}, \mathrm{v}_{9}\right)=0 ; \operatorname{dom}\left(\mathrm{v}_{1}, \mathrm{v}_{10}\right)=0 ; \operatorname{dom}$ $\left(\mathrm{v}_{1}, \mathrm{v}_{11}\right)=0 ; \operatorname{dom}\left(\mathrm{v}_{1}, \mathrm{v}_{12}\right)=0 ;$ dom $\left(\mathrm{v}_{1}, \mathrm{v}_{13}\right)=0 ;$ dom $\left(\mathrm{v}_{1}, \mathrm{v}_{14}\right)=0 ; \operatorname{dom}\left(\mathrm{v}_{2}, \mathrm{v}_{3}\right)=1 ; \operatorname{dom}\left(\mathrm{v}_{2}, \mathrm{v}_{4}\right)=1 ; \operatorname{dom}\left(\mathrm{v}_{2}, \mathrm{v}_{5}\right)$ $=0 ; \operatorname{dom}\left(\mathrm{v}_{2}, \mathrm{v}_{6}\right)=1 ; \operatorname{dom}\left(\mathrm{v}_{2}, \mathrm{v}_{7}\right)=0 ; \operatorname{dom}\left(\mathrm{v}_{2}, \mathrm{v}_{8}\right)=0$ ;dom $\left(\mathrm{v}_{2}, \mathrm{v}_{9}\right)=0 ; \operatorname{dom}\left(\mathrm{v}_{2}, \mathrm{v}_{10}\right)=0 ; \operatorname{dom}\left(\mathrm{v}_{2}, \mathrm{v}_{11}\right)=0 ;$ dom $\left(\mathrm{v}_{2}, \mathrm{v}_{12}\right)=0 ; \operatorname{dom}\left(\mathrm{v}_{2}, \mathrm{v}_{13}\right)=0 ; \operatorname{dom}\left(\mathrm{v}_{2}, \mathrm{v}_{14}\right)=0 ;$ dom $\left(\mathrm{v}_{3}, \mathrm{v}_{4}\right)=1 ; \operatorname{dom}\left(\mathrm{v}_{3}, \mathrm{v}_{5}\right)=1 ; \operatorname{dom}\left(\mathrm{v}_{3}, \mathrm{v}_{6}\right)=0 ; \operatorname{dom}\left(\mathrm{v}_{3}, \mathrm{v}_{7}\right)=$ $0 ; \operatorname{dom}\left(\mathrm{v}_{3}, \mathrm{v}_{8}\right)=0 ; \operatorname{dom}\left(\mathrm{v}_{3}, \mathrm{v}_{9}\right)=0 ; \operatorname{dom}\left(\mathrm{v}_{3}, \mathrm{v}_{10}\right)=0$; $\operatorname{dom}\left(\mathrm{v}_{3}, \mathrm{v}_{11}\right)=0 ; \operatorname{dom}\left(\mathrm{v}_{3}, \mathrm{v}_{12}\right)=0 ; \operatorname{dom}\left(\mathrm{v}_{3}, \mathrm{v}_{13}\right)=0 ; \operatorname{dom}$ $\left(\mathrm{v}_{3}, \mathrm{v}_{14}\right)=0$;

$\operatorname{dom}\left(\mathrm{v}_{4}, \mathrm{v}_{5}\right)=1 ; \operatorname{dom}\left(\mathrm{v}_{4}, \mathrm{v}_{6}\right)=1 ; \operatorname{dom}\left(\mathrm{v}_{4}, \mathrm{v}_{7}\right)=0 ; \operatorname{dom}$ $\left(\mathrm{v}_{4}, \mathrm{v}_{8}\right)=0 ; \operatorname{dom}\left(\mathrm{v}_{4}, \mathrm{v}_{9}\right)=0 ; \operatorname{dom}\left(\mathrm{v}_{4}, \mathrm{v}_{10}\right)=1 ;$

$\operatorname{dom}\left(\mathrm{v}_{4}, \mathrm{v}_{11}\right)=0 ; \operatorname{dom}\left(\mathrm{v}_{4}, \mathrm{v}_{12}\right)=0 ; \operatorname{dom}\left(\mathrm{v}_{4}, \mathrm{v}_{13}\right)=0$ $; \operatorname{dom}\left(\mathrm{v}_{4}, \mathrm{v}_{14}\right)=0 ; \operatorname{dom}\left(\mathrm{v}_{5}, \mathrm{v}_{6}\right)=1 ; \operatorname{dom}\left(\mathrm{v}_{5}, \mathrm{v}_{7}\right)=1 ; \operatorname{dom}$ $\left(\mathrm{v}_{5}, \mathrm{v}_{8}\right)=0 ; \operatorname{dom}\left(\mathrm{v}_{5}, \mathrm{v}_{9}\right)=1 ; \operatorname{dom}\left(\mathrm{v}_{5}, \mathrm{v}_{10}\right)=1 ; \operatorname{dom}\left(\mathrm{v}_{5}, \mathrm{v}_{11}\right)$ $=0 ; \operatorname{dom}\left(\mathrm{v}_{5}, \mathrm{v}_{12}\right)=0 ; \operatorname{dom}\left(\mathrm{v}_{5}, \mathrm{v}_{13}\right)=0 ; \operatorname{dom}\left(\mathrm{v}_{5}, \mathrm{v}_{14}\right)=0 ;$ $\operatorname{dom}\left(\mathrm{v}_{6}, \mathrm{v}_{7}\right)=1 ; \operatorname{dom}\left(\mathrm{v}_{6}, \mathrm{v}_{8}\right)=1 ; \operatorname{dom}\left(\mathrm{v}_{6}, \mathrm{v}_{9}\right)=0 ; \operatorname{dom}$ $\left(\mathrm{v}_{6}, \mathrm{v}_{10}\right)=1 ; \operatorname{dom}\left(\mathrm{v}_{6}, \mathrm{v}_{11}\right)=0 ; \operatorname{dom}\left(\mathrm{v}_{6}, \mathrm{v}_{12}\right)=0 ; \operatorname{dom}$ $\left(\mathrm{v}_{6}, \mathrm{v}_{13}\right)=0 ; \operatorname{dom}\left(\mathrm{v}_{6}, \mathrm{v}_{14}\right)=0 ; \operatorname{dom}\left(\mathrm{v}_{7}, \mathrm{v}_{8}\right)=1 ; \operatorname{dom}\left(\mathrm{v}_{7}, \mathrm{v}_{9}\right)$ $=1 ; \operatorname{dom}\left(\mathrm{v}_{7}, \mathrm{v}_{10}\right)=0$;

$\operatorname{dom}\left(\mathrm{v}_{7}, \mathrm{v}_{11}\right)=1 ; \operatorname{dom}\left(\mathrm{v}_{7}, \mathrm{v}_{12}\right)=0 ; \operatorname{dom}\left(\mathrm{v}_{7}, \mathrm{v}_{13}\right)=0 ; \operatorname{dom}$ $\left(\mathrm{v}_{7}, \mathrm{v}_{14}\right)=0 ; \operatorname{dom}\left(\mathrm{v}_{8}, \mathrm{v}_{9}\right)=1 ; \operatorname{dom}\left(\mathrm{v}_{8}, \mathrm{v}_{10}\right)=1 ;$

$\operatorname{dom}\left(\mathrm{v}_{8}, \mathrm{v}_{11}\right)=1 ; \operatorname{dom}\left(\mathrm{v}_{8}, \mathrm{v}_{12}\right)=1 ; \operatorname{dom}\left(\mathrm{v}_{8}, \mathrm{v}_{13}\right)=0 ; \operatorname{dom}$ $\left(\mathrm{v}_{8}, \mathrm{v}_{14}\right)=1 ; \operatorname{dom}\left(\mathrm{v}_{9}, \mathrm{v}_{10}\right)=1 ; \operatorname{dom}\left(\mathrm{v}_{9}, \mathrm{v}_{11}\right)=1 ;$ dom $\left(\mathrm{v}_{9}, \mathrm{v}_{12}\right)=0 ; \operatorname{dom}\left(\mathrm{v}_{9}, \mathrm{v}_{13}\right)=1 ; \operatorname{dom}\left(\mathrm{v}_{9}, \mathrm{v}_{14}\right)=1 ; \operatorname{dom}\left(\mathrm{v}_{10}, \mathrm{v}_{11}\right)$ $=0 ; \operatorname{dom}\left(\mathrm{v}_{10}, \mathrm{v}_{12}\right)=0 ; \operatorname{dom}\left(\mathrm{v}_{10}, \mathrm{v}_{13}\right)=0 ; \operatorname{dom}\left(\mathrm{v}_{10}, \mathrm{v}_{14}\right)=$ $1 ; \operatorname{dom}\left(\mathrm{v}_{11}, \mathrm{v}_{12}\right)=1 ; \operatorname{dom}\left(\mathrm{v}_{11}, \mathrm{v}_{13}\right)=1 ; \operatorname{dom}\left(\mathrm{v}_{11}, \mathrm{v}_{14}\right)=0 ;$ dom $\left(\mathrm{v}_{12}, \mathrm{v}_{13}\right)=1 ; \operatorname{dom}\left(\mathrm{v}_{12}, \mathrm{v}_{14}\right)=1$;

$\operatorname{dom}\left(\mathrm{v}_{13}, \mathrm{v}_{14}\right)=1$.

\section{$T D V(G)=38$.}

Medium Domination Number $=\frac{2(38)}{14(13)}=\frac{38}{91}$.

THEOREM 3.4: The Medium Domination Number of Linear Polyacene $\boldsymbol{L}_{\boldsymbol{h}}$ is

$$
\operatorname{MD}\left(\boldsymbol{L}_{\boldsymbol{h}}\right)=2 \sum_{k=1}^{h} \frac{13 k-1}{(4 k+2)(4 k+1)} .
$$

Proof

Let $\boldsymbol{L}_{\boldsymbol{h}}$ be Linear Polyacene with h Hexagones.

It is enough to show that the Medium Domination Number of Linear Polyacene $\boldsymbol{L}_{\boldsymbol{h}}$ is

$\operatorname{MD}\left(\boldsymbol{L}_{\boldsymbol{h}}\right)=2 \sum_{k=1}^{h} \frac{13 k-1}{(4 k+2)(4 k+1)}$

by Induction.

Step 1:

Put $\mathrm{h}=1$ in equation(I), we get

$\boldsymbol{L}_{\boldsymbol{l}}$-Linear Polyacene with one Hexagone(Benzene).

There are 6 vertices $\mathrm{v}_{1}, \mathrm{v}_{2}, \mathrm{v}_{3}, \mathrm{v}_{4}, \mathrm{v}_{5}, \mathrm{v}_{6}$ (Figure 1$)$.

By 3.1, The summation of vertices that dominate every set of two vertices is 12 .

Therefore, By Definition 2.6, the Medium Domination Number of Linear Polyacene $\boldsymbol{L}_{\boldsymbol{l}}$ is

$\operatorname{MD}\left(L_{1}\right)=\frac{2(12)}{6(5)}=\frac{4}{5}$

Published By:

Blue Eyes Intelligence Engineering

\& Sciences Publication 
Step 2:

Put $\mathrm{h}=2$ in equation(I), we get

$\boldsymbol{L}_{2}$ - Linear Polyacene with two Hexagons(Naphthalene).

By 3.2, The summation of vertices that dominate every set of two vertices is 25 .

Therefore, By Definition 2.6 and 3.2, the Medium Domination Number of Linear

Polyacene $\boldsymbol{L}_{2}$ is $\operatorname{MD}\left(\boldsymbol{L}_{2}\right)=\frac{2(25)}{\mathbf{1 0}(9)}=\frac{\mathbf{5}}{9}$.

Assume that $\boldsymbol{L}_{\boldsymbol{h}-1}$ is Linear Polyacene with $h-1$ Hexagons where $h \geq 2 \in N$.

Then the Medium Domination Number of Linear Polyacene $\boldsymbol{L}_{\boldsymbol{h}-\boldsymbol{I}}$ is

$$
\operatorname{MD}\left(L_{h-1}\right)=2 \sum_{i=2}^{h} \frac{13 i-1}{(4 i+2)(4 i+1)} .
$$

We have to show $\operatorname{MD}\left(\boldsymbol{L}_{\boldsymbol{h}}\right)=2 \sum_{i=1}^{h} \frac{13 i-1}{(4 i+2)(4 i+1)}$ for $\boldsymbol{L}_{\boldsymbol{h}}$, Linear Polyacene with $h$ Hexagones

where $h \geq 1 \in \mathrm{N}$.

By hypothesis, summation of vertices that dominate every set of two vertices is $13 h-1$

for $h$ hexagones.

By step 1, summation of vertices that dominate every set of two vertices is 12 .

Now, the summation of vertices that dominate every set of two vertices for $h$ hexagons is equal to the sum of total number of vertices that every set of two vertices for one hexagon and total number of vertices that dominate every set of two vertices for $h-1$ hexagons

where $h \geq 2$.

$$
\begin{aligned}
& \text { Hence } \operatorname{MD}\left(L_{h}\right)=\frac{2(12)}{6(5)}+2 \sum_{i=2}^{h} \frac{13 i-1}{(4 i+2)(4 i+1)} \\
& =2\left\{\frac{13(1)-1}{(4(1)+2)(4(1)+1)}+\sum_{i=2}^{h} \frac{13 i-1}{(4 i+2)(4 i+1)}\right\} \\
& =2 \sum_{i=1}^{h} \frac{13 i-1}{(4 i+2)(4 i+1)} .
\end{aligned}
$$

By Induction Principle, $\operatorname{MD}\left(L_{h}\right)=2 \sum_{k=1}^{h} \frac{13 k-1}{(4 k+2)(4 k+1)}$ for $h$ Hexagons where $h \geq 1 \in N$.

\section{CONCLUSION}

In this Paper, the Molecular Graph such as Benzene, Naphthalene and Anthracene obtained Medium Domination Number for them. It also explained Medium Domination Number of Linear Polyacene $\boldsymbol{L}_{\boldsymbol{h}}$ is $\operatorname{MD}\left(\boldsymbol{L}_{\boldsymbol{h}}\right)=2$ $\sum_{k=1}^{h} \frac{13 k-1}{(4 k+2)(4 k+1)}$ by Induction method.

\section{REFERENCES}

1. S.Arumugam, Invitation to Graph Theory, Scitech Publications(India) Pvt Limited, ISBN 139788187328469, 2014.

2. Duygu Vargorn and Pinar Dundar, The Medium Domination Number Of a Graph, International Journal of Pure and Applied Mathematics, Vol.70 No.3,297-306,(2011).

3. M. Ramachandran and N. Parvathi, The Medium Domination Number Of a Jahangir Graph $\mathbf{J}_{\mathrm{m}, \mathrm{n}}$, Indian Journal of Science and Technology, Vol.8(5),400-406,(2015).

4. S.Vijayalakshmi, M.Raji and G.Jayalalitha, Degree based in Molecular Graph of Organic compounds on Domination, Journal of Advanced Research in Dynamical and Control Systems, Vol.10, 06-special issue, 962-966,(2018).

5. G.Jayalalitha, M.Raji, S.Senthil, Hosoya Polynomial and Wiener Index of Molecular Graph of Naphthalene Based On
Domination, International Journal of Scientific Research and Review, Vol.8,Issue 1, 1238-1241,(2019).

6. Nisreen Bukhary, Domination in Benzenoids. Virginia Commonwealth University,VCU Scholars Compass, 2010.

7. Sandi Klavzar, Ivan Gutman , A Comparison of the Schultz Topological Index with the Wiener Index, Journal for Chemical Information and Computer Sciences, 36, 10011003,(1996).

8. Nenad Trinajstic,Chemical Graph Theory,Second Edition,CRC Press, 1983. 